\title{
Path-oriented early reaction to approaching disruptions in ASDEX Upgrade and TCV in view of the future needs for ITER and DEMO
}

\author{
M.Maraschek ${ }^{1}$, A.Gude ${ }^{1}$, V.Igochine ${ }^{1}$, H.Zohm ${ }^{1}$, \\ E.Alessi $^{3}$, M.Bernert ${ }^{1}$, C.Cianfarani ${ }^{4}$, S.Coda ${ }^{2}$, B.Duval ${ }^{2}$, B.Esposito ${ }^{4}$, S.Fietz $^{1}$, M.Fontana ${ }^{2}$, \\ C.Galperti ${ }^{2}$, L.Giannone ${ }^{1}$, T.Goodman ${ }^{2}$, G.Granucci ${ }^{3}$, L.Marelli ${ }^{5}$, S.Novak ${ }^{3}$, R.Paccagnella ${ }^{5}$, \\ G.Pautasso $^{1}$, P.Piovesan ${ }^{5}$, L.Porte ${ }^{2}$, S.Potzel ${ }^{1}$, C.Rapson ${ }^{1}$, M.Reich $^{1}$, O.Sauter ${ }^{2}$ U.Sheick $^{2}$, \\ C.Sozzi $^{3}$, G.Spizzo ${ }^{5}$, J.Stober ${ }^{1}$, P.Zanca ${ }^{5}$, ASDEX Upgrade team, TCV team, the EUROfusion \\ MST1 Team $^{6}$ \\ 1 Max-Planck-Institut for Plasmaphysics, D-85748 Garching, Germany \\ 2 Ecole Polytechnique Fédérale de Lausanne (EPFL), Swiss Plasma Center (SPC), 1015, Switzerland \\ 3 IFP-CNR, via R. Cozzi 53, 20125 Milano, Italy \\ 4 ENEA, Fusion and Nuclear Safety Department, C. R. Frascati, 00044 Frascati (Roma), Italy \\ 5 Consorzio RFX, corso Stati Uniti 4, 35127 Padova, Italy \\ ${ }^{6}$ see the author list in [H. Meyer et al., Nuclear Fusion FEC 2016 Special Issue (2017)] \\ E-mail: Maraschek@ipp.mpg.de
}

PACS number: 52.55.Fa,52.55.Tn,28.52.Cx,28.52.Cx

Keywords: tokamak, disruption avoidance, ECCD, plasma-state, density limit, control

\begin{abstract}
Routine reaction to approaching disruptions in tokamaks is currently largely limited to machine protection by mitigating an ongoing disruption, which remains a basic requirement for ITER and DEMO [1]. Nevertheless, a mitigated disruption still generates stress to experiment. Additionally, in future fusion devices, high-performance discharge time itself will be very valuable. Instead of reacting only on generic features, occurring shortly before the disruption, the ultimate goal is to actively avoid approaching disruptions at an early stage, sustain the discharges whenever possible and restrict mitigated disruptions to major failures. Knowledge of the most relevant root causes and the corresponding chain of events leading to disruption, the disruption path, is a prerequisite. For each disruption path, physics-based sensors and adequate actuators must be defined and their limitations considered. Early reaction facilitates the efficiency of the actuators and enhances the probability of a full recovery. Thus, sensors that detect potential disruptions in time are to be identified.

Once the entrance into a disruption path is detected, we propose a hierarchy of actions consisting of (I) recovery of the discharge to full performance or at least continuation with a less disruption-prone backup scenario, (II) complete avoidance of the disruption to sustain the discharge or at least delay it for a controlled termination and, (III), only as last resort, a disruption mitigation. Based on the understanding of disruption paths, a hierarchical and path-specific handling strategy must be developed. Such schemes, testable in present devices, could serve as guideline for ITER and DEMO operation.

For some disruption paths, experiments have been performed at ASDEX Upgrade and TCV. Disruptions were provoked in TCV by impurity injection into ELMy H-mode discharges and in ASDEX Upgrade by reaching the density limit both in L and H-modes. The new approach proposed in this paper is discussed in some detail for these cases. Sensors used so far react too late for preventing an H-mode density limit. A plasma-state boundary is proposed, that can serve as an adequate early sensor for avoiding density limit disruptions in H-modes and for recovery to full performance.
\end{abstract}

\section{Introduction}

Disruptions are a major concern for large tokamaks, as the wall heat load by the thermal energy quench, the electromagnetic forces generated during the current quench and the formation of an electron runaway beam cause problems. Present experiments mainly focus on reducing these thermal and mechanical loads by mitigating the disruption. This involves the injection of radiating impurities that reduce the plasma energy and speed up the current decay, while an elongated plasma might undergo a vertical shift. This thermal load and force mitigation method is also applicable to ITER [2]. However, it is not known whether and how the required densities for an avoidance of an electron runway beam can be reliably achieved in ITER.

Such mitigated discharges nevertheless disrupt and still cause thermal and mechanical loads and 
possibly generate runaway electrons. In future devices, such as ITER and DEMO, disruption mitigation must therefore be restricted to the unavoidable cases. Additionally, discharge time is valuable, such that a premature termination of a running discharge, even by a controlled ramp-down, has to be avoided, if a continuation is possible without risking damage. With these more demanding requirements compared to presently implemented control schemes, the discharge control and the handling of near-disruption states must be reconsidered. This requires significantly earlier detection of an approaching disruption than is achievable by detecting generic common features appearing typically only shortly before a disruption. We propose a concept which could be implemented already in present devices. The gained experience should support the future operation of ITER and DEMO.

The paper is structured as follows. In section 2, the present status of experiments and routine handling of disruption, including sensors and actuators, is briefly indicated. In section 3, a new generalized approach on disruption handling is proposed. In section 4 and 5, the latest experiments at TCV and ASDEX Upgrade are described and how they fit in our concept. Finally, in section 6 , the present results are summarized and the envisaged next steps are indicated.

\section{Disruption types and their handling}

To better understand and handle disruptions, the root causes of disruptions were analyzed for JET with carbon facing components [3]. Later, analysis of operation with the ITER-like wall (tungsten and beryllium) at JET [4] and of ASDEX Upgrade (AUG) with its tungsten wall [5] was performed. Based on these classifications the most important types of disruptions and their causes were extracted (see table 1). Each type of disruption follows a sequence of events, i.e. its path, path $_{i}$, until the discharge disrupts. Such paths will serve as the physics basis for early disruption handling. In the following subsection we briefly describe the most relevant disruption paths.

\subsection{Paths leading to disruptions}

In the ideal or resistive high $\beta_{N}$ limit, the pressure and hence its gradient at resonant surfaces increases to a value where ideal or resistive MHD limits are reached and a possibly disruptive MHD mode can be triggered. In the resistive case a Neoclassically driven Tearing Mode (NTM) gets triggered, which is driven by the loss of bootstrap current within the island's O-point. Depending on the discharge scenario, faster growing ideal modes can be triggered at higher $\beta_{N}$. Here the ideal kink mode and the resistive wall mode (RWM) play the crucial role.

\begin{tabular}{|l|l|}
\hline root cause & description \\
\hline$\beta_{N}$ limit & $\begin{array}{l}\text { pressure driven resistive or ideal } \\
\text { core MHD modes, mode locking }\end{array}$ \\
\hline L-mode $n_{e}$ limit & $\begin{array}{l}\text { edge limit, divertor detachment, } \\
\text { MARFE, } T_{e} \text { and } I_{p} \text { profile } \\
\text { changes, current driven modes, } \\
\text { mode locking }\end{array}$ \\
\hline H-mode $n_{e}$ limit & $\begin{array}{l}\text { edge limit, H-L transition, diver- } \\
\text { tor detachment, MARFE, late } \\
\text { current driven modes, mode } \\
\text { locking }\end{array}$ \\
\hline $\begin{array}{l}\text { high-Z impurity } \\
\text { accumulation }\end{array}$ & $\begin{array}{l}\text { metal wall issue, radiation peak- } \\
\text { ing and losses, resistive MHD } \\
\text { mode in the core, mode locking }\end{array}$ \\
\hline technical failure & $\begin{array}{l}\text { irregular influx or drop of mate- } \\
\text { rial or debris, control system or } \\
\text { power supply failure, ... }\end{array}$ \\
\hline
\end{tabular}

Table 1. List of most important disruption types identified from AUG and JET $[3,4,5]$.

In the density limit (DL), both in L-mode and $\mathrm{H}$-mode, the plasma is strongly cooled from the edge by a high particle influx. Ultimately in the L-mode, in both cases a X-point radiator, also named MARFE (Multi-faceted Assymetric Radiation From the Edge) [6], is formed. The edge cooling modifies temperature, density and in particular the current profiles increasing the likelihood of MHD activity. Typically tearing modes with toroidal mode number $n=1$ are initiated and, once toroidally coupled with different poloidal mode numbers, $m$, over multiple resonant surfaces, lead to a disruption when they finally lock to the wall. These modes are thus not the disruption root cause, but a late result of the discharge development.

In the $\mathrm{H}$-mode DL edge cooling first leads to an $\mathrm{H}$ $\mathrm{L}$ transition and finally to a DL disruption in L-mode $[7,8,9]$. In [9] the H-L transition is described in terms of the stored energy $W_{m h d}$ and a line integrated edge density. This is an example of a state-space description of the plasma, which will be discussed in detail in section 5 with the corresponding experiments.

In experiments with metallic walls (for example AUG with its tungsten wall or JET with an ITER-like wall with a combination of tungsten and beryllium), high-Z impurities can enter the plasma. Owing to neoclassical inward transport, they tend to accumulate in the plasma core and act there as a strong radiator, as they are not fully ionized. Excessive core radiation cools the plasma and generates hollow temperature profiles, which makes the discharge unstable and initiates the disruption $[10,11]$.

A common feature for most paths is the occurrence 
of $(m \geq 2 / n=1)$ modes, which lock to the wall and ultimately lead to a disruption.

Occasionally, a Vertical Displacement Event (VDE) occurs, which is a loss of the control over the vertical position of the plasma. It can be caused by a too fast movement of the plasma or a technical failure.

Additional to these operational limits related to plasma stability, technical failures may occur. These are either mechanical in-vessel failures leading to a release of impurities. or debris entering the confined plasma region. Depending on the severity, this can produce various chains of events that lead to a final disruption. On the other hand, the control system or the power supply of the experiment may fail and measures have to be foreseen, to handle such events.

\subsection{Sensors for early disruption detection}

The identification of an approaching disruption and the proximity to its operational boundary must be obtained at the earliest reasonable time point to start an appropriate action. For each of the considered paths a dedicated set of sensors is required, which are not necessarily simple direct measurements, but might be complex combinations of signals describing the plasma state in a path-specific state space. The sensors must be available during the discharge in real time and reliably detect the relevant plasma parameters. Table 2 gives an indication of possible disruption sensors which fulfil these conditions.

\begin{tabular}{|l|cll|}
\hline sensor & \multicolumn{2}{|c|}{ action } \\
\hline rotating mode detector & I & II & \\
\hline locked mode detector & & II & III \\
\hline loop voltage & & II & III \\
\hline VDE detector & & III \\
\hline $\begin{array}{l}\text { MARFE detectors (bolometer, } \\
\text { bremsstrahlung, detachment via }\end{array}$ & & & \\
$H_{\alpha}$ II the divertor) & & & \\
\hline$n_{e}$ and radiation peaking (interfer- & I & (II) & \\
ometer, bolometer, soft X-ray) & & & \\
\hline
\end{tabular}

Table 2. List of individual sensors and for which type of action they are mostly relevant. The actions are defined in section 3.1.

In particular, in the high $\beta_{N}$ limit strong ideal or resitive MHD modes occur. The MHD modes in the density limit are occurring late in the L-mode phase of the disruption. Various detection methods exist for (still) rotating MHD modes. To identify the potential threat of specific modes, its rotation frequency, amplitude, radial location and the toroidal and poloidal mode numbers, $n$ and $m$, are relevant parameters. The mode numbers can be obtained through the analysis of a spatially distributed set of Mirnov coils, that measure the perturbation field.
Techniques that employ individual coils only [12] rely upon specific assumptions and are thus not applicable as safe disruption sensor. Recently, a real-time method, based on singular value decomposition (SVD) analysis of Mirnov coil signals, was implemented at FTU, and ported to AUG and TCV [13]. It calculates the entropy, $H$, of the SVD eigenvalue distribution which is a measure for the degree of correlation. $H$ typically decreases well before the amplitude of the corresponding perturbation field rises above the noise level. In combination with the provided likelihoods for multiple $(m / n)$ combinations, the identification of mode numbers is possible already for low amplitude modes. Real-time correlation analysis of a spatially filtered magnetic signal with measurements of the local electron temperature (ECE), provides the radial location and the eigenfunction of the modes [14].

The commonly occurring LMs can in principle be easily detected by a linear combination of at least 4 toroidally distributed saddle coils to obtain the perturbation field amplitude, $B_{r}^{\text {pert }}$, and its phase [15]. Large LMs typically occur only very shortly before the disruption and are mainly useful for triggering mitigation actions. Generally, LMs with intermediate size can also be used for disruption avoidance.

Typically, large perturbations in the plasma, such as large MHD modes, increasing radiation losses or current profile peaking, lead to an increase of the required loop voltage, $U_{\text {loop }}$, to maintain the requested plasma current, $I_{p}$. Either a fixed pre-defined or a dynamical adaptable threshold on $U_{\text {loop }}$ serves as a trigger for reactions of the control system. The $U_{l o o p}$ rise is also typical for density limits and can be used for avoidance and mitigation.

The VDE detector is implemented by a real-time comparison between the requested and the observed vertical position of the plasma. At AUG an immediate mitigation reaction is triggered when control is no longer feasible for a deviation larger than a pre-defined technical threshold.

The MARFE occurring in density limits, is detected through a tomographic inversion of a bolometer array. With increasing computing power, suitable algorithms are able to detect the MARFE formation and its radial and vertical location in real time [16]. In previous experiments also the divertor detachment, detected from the $H_{\alpha}$ emission of the inner and outer divertor, has been used as a measure of the degree of detachment and hence the operational proximity of MARFE formation in AUG. The MARFE itself was detected from the X-point emission of bremsstrahlung [17].

In the high-Z impurity induced path the increase of the core plasma radiation together with its peaking is a clear indicator for proximity to a disruption. This 
is calculated from several lines of sight of a bolometry or soft X-ray system.

The combination of multiple measurements allows the definition of a plasma-state description, as it is done for example in the RAPTOR code [18]. Such states are able to characterize the proximity to operational boundaries of the plasma, which can be used to start the reaction on approaching disruptions. In section 5 we will present an operational boundary in its corresponding state space description for the $\mathrm{H}$ mode density limit based on a generalized $\overline{n_{e}}-W_{m h d}$ diagram [9]. Together with a dynamical model of the plasma, but without dedicated models for the disruption paths, a prediction of the non-disruptive discharge development can be made. A deviation of the observed plasma state from the prediction provides a trigger indicating the discharge does not develop according to the prescribed route. Including models for the disruption paths itself would provide a direct path-specific indicator to start appropriate actions.

Recently, for high $\beta_{N}$ disruptions induced by RWMs in NSTX, a stability analysis has been performed based on a reduced kinetic MHD model $[19,20]$. This approach uses the corresponding MHD stability as boundary for initiating early disruption avoidance schemes, which aim to operate the discharge without reaching the limit and triggering an RWM. The operation point in the stability diagram of the RWM corresponds to a plasma state and the crossing of the stability boundary serves as trigger.

\subsection{Actuators for disruption handling}

Once an approaching disruption along a specific path has been detected with its corresponding physics-based disruption identifiers, a set of possible actuators is available to deal with the situation. Table 3 lists actuators, which are presently used and also considered for the future. This list is still incomplete. In this subsection we discuss the applicability of these actuators for specific paths.

In high $\beta_{N}$ scenarios where ideal or resistive MHD modes play the dominant role for the disruption (i.e. in the high $\beta_{N}$-limit) local ECCD current drive at the resonant surface of the MHD mode is the most appropriate tool. For the resistive case, various experiments for the stabilization of the $(2 / 1)$ NTMs have been performed [21, 22, 23, 24, 25, 26]. The additional combination with external resonant magnetic perturbations (RMP) allows to control the island phase and steer the ECCD, now modulated, only into the O-point of the island, i.e. only inside the separatrix of the island $[25,27,28]$. RMPs can also be used to avoid the locking of an MHD mode by compensating the intrinsic error field or entrain the mode to a rotating perturbation field[27, 28]. However, effective RMP application requires sufficiently low electron density for the penetration of the perturbation field up to the resonant surface, and sufficiently high $\beta_{N}$ to take advantage of resonant field amplification [29]. Both ECRH/ECCD and RMPs will be available for ITER and DEMO, yet the applicability of the RMPs in this context has to be further investigated.

Both in the $\mathrm{L}$ and $\mathrm{H}$-mode density limit $n=1$ modes occur that play the key role in the final thermal and current quench. These modes can be addressed in the same way as NTMs by local heating or current drive at the resonant surface. Applying in an Ohmic L-mode one central gyrotron for heating and one gyrotron for current drive at the $q=2$ surface triggered by a LM or $U_{\text {loop }}$, the electron density could be raised by nearly a factor of 2 above the level at which the disruption set in $[30]$.

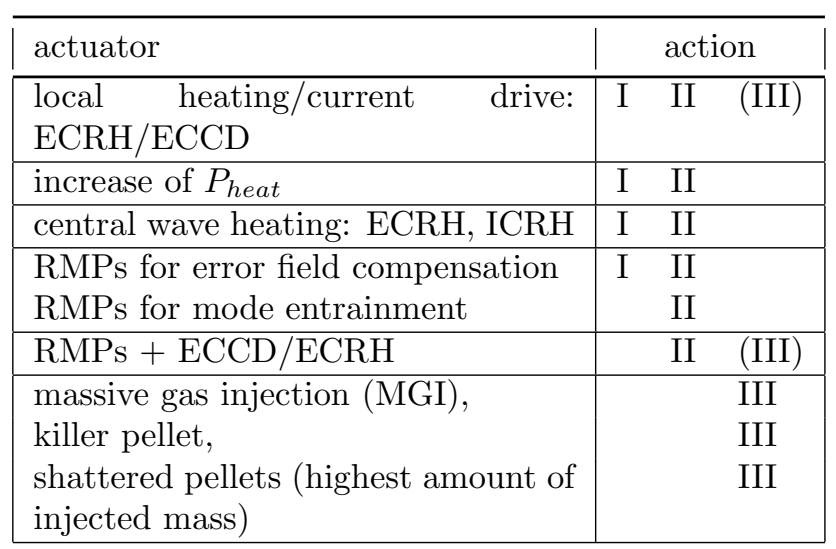

Table 3. List of actuators and for which type of action they are mostly relevant. The actions are defined in section 3.1.

Alternatively, radiation losses near the density limit can be counteracted with additional heating power. This was successfully and routinely done with additional NBI heating on AUG, which was triggered by the divertor detachment sensor [17], and with additional central ICRH, as it has been demonstrated on ADITYA [31]. At AUG, the use of additional NBI heating power and a control of the applied gas influx was used routinely to avoid density limit disruptions. However, such approaches will have to compete with the loss of $\alpha$-particle heating in future devices.

For high $\mathrm{Z}$ impurity accumulation in devices with metal walls, the most important actuator is central heating without additional central particle fuelling. At AUG and JET this is achieved by central wave heating ( $\rho_{p} \approx 0$ within the $q=1$ surface) with ECRH or ICRH [32]. This application routinely stabilizes discharges on both devices and will also be available for the operation of ITER. However, in ITER the central heating will be dominated by the $\alpha$-particles, which non-linearly depends on the plasma conditions. It is not yet clear if the external heating will be able to replace a possible 
loss of the $\alpha$-heating. Both heating systems will be available in future devices.

For disruption mitigation, the deliberate massive density increase is employed in order to radiate as much energy as possible, for cases where a disruption is considered inevitable. A massive increase in density also suppresses a possible runaway electron beam [33]. At AUG, this mitigation is used routinely for machine protection by a dedicated massive gas injection (MGI) [34]. Injection of killer pellets with impurities has been tested [35, 36], and still remains an option for the future. Recently, shattered pellet injection (SPI) has been established in DIII-D [37] providing the largest amount of deposited material within these methods. Experiments with SPI are presently prepared at JET for 2018 and will provide input for the dissipation of the runaway current, and provides input for the foreseen SPI system at ITER [2],

However, most of the attempts to avoid disruptions are merely proof of principle experiments for the specific discharge scenario. A combination of the sensors and actuators in an automatic protection scheme has yet to be implemented. The operational experience is urgently required for future experiments.

\subsection{Present routine disruption handling}

On present devices, only a very limited number of sensors and actuators are routinely used. At AUG, depending on the level of the locked mode signal, either a controlled ramp-down of the discharge or an MGI for mitigation $\left(I_{p}>0.85 \mathrm{MA}\right)$ is triggered. For a VDE, detected by the control system, the MGI is triggered instantaneously. Following technical failures of the control system or the power supplies, the MGI is also initiated.

At JET, the $n=2$ trigger initiates a soft-stop trying to safely ramp down the discharge with full control. The locked mode (LM) trigger initiates, dependend on the plasma current, a fast stop, which tries to immediately bring the experiment in a safe condition also accepting a mitigated disruption. When an ongoing disruption is identified by a sharp drop in $I_{p}\left(d I_{p} / d t>\left(d I_{p} / d t\right)_{\text {trigger }}\right)$ MGI is always applied for mitigation. The automatic MGI trigger from the LM detector has become a necessity for the operation with the ITER like wall [11]. Additionally to these pre-programmed reactions a framework of conditions allows the session leader to define reactions via a set of hierarchical segment branching. This has to be set up individually for a specific discharge and is not necessarily routinely active.

The DIII-D plasma control system (PCS), used also at EAST and KSTAR [38], contains various event driven reactions and possibilities to activate different waveforms in real time [39]. It contains also sophisiticated event handling schemes, which are also applied to disruption prevention and mitigation $[40,41]$.

\section{Generalized approach}

The above indicated concepts for identifying disruption categories and connecting them to their path $_{i}$ (such as $\beta_{N}$-limit, path $_{\beta_{N}}$, the L-mode density limit, path $h_{L D L}$, or the H-mode density limit, path $h_{H D L}$ ) have to be combined into an event handling framework, which has to become an integral part of the control system. (Plasma Control System (PCS)). Such a framework could be implemented in present experiments [39, 40] and is foreseen for the ITER control system [40, 42, 43, 41]. At DIII-D similar approaches, as we will discus below, are considered and implemented [44].

\subsection{Categorization of actions}

The action which has to be applied to the plasma will necessarily be case dependent. It will not only depend on the path $_{i}$, but also the severity of the situation has to be taken into account for the selection of the required action. We suggest the following hierarchical actions, which have to be defined for each path $_{i}$ individually.

I recovery of the discharge to full performance or continuation with a less disruption-prone backup

II complete avoidance of an ongoing disruption to sustain the discharge or delay it for a controlled shutdown

\section{III mitigation of an inevitable disruption}

In present dedicated disruption experiments the path is pre-selected by the type of discharge which is performed. For each experimental setup, the sensors, sensor $_{i, j}$, are manually selected and combined. For the action, action $_{i, k}(k \in\{I, I I, I I I\})$ a set of actuators, $\Sigma_{k, m}$ actuator $_{i, k, m}$, can be used. Tables 2 and 3 summarize a list of sensors and actuators and for which of the actions they are considered as most relevant.

This concept is illustrated for the high $\beta_{N}$-limit, where pressure driven resistive MHD plays the main role. The removal of rotating (3/2)-NTMs with local ECCD at the resonant surface of the mode can be understood as an early recovery scheme. Either full plasma performance is re-established or a branch towards a low $\beta_{N}$ program is possible. The removal of the more dangerous $(m \geq 2 / n=1)$-NTMs and the unlocking of a locked $n=1$ mode using ECCD corresponds primarily to disruption avoidance. For unlocking RMPs are an additional actuator. In case of a larger amplitude $n=1$ locked mode disruption mitigation becomes necessary. 


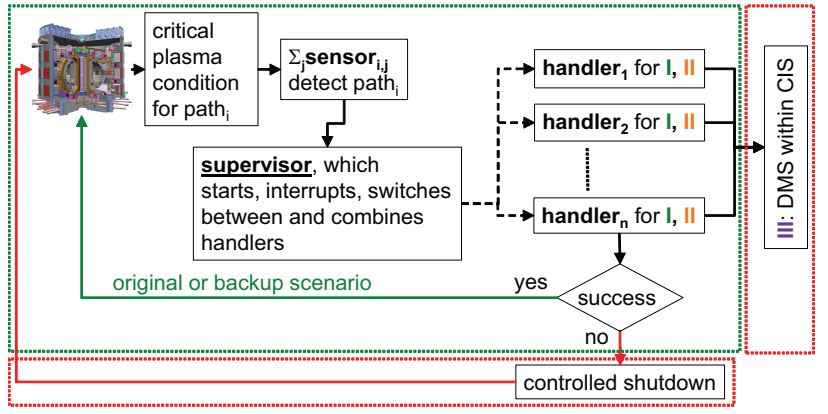

Figure 1. Schematic flow diagram of the outer control loop, containing for each path $_{i}$ its corresponding handler (see figure 2), a supervisor reacting on identified paths starting, interrupting, exchanging or combining handlers. The green area is the desired operational area, whereas the red area (unwanted controlled shutdown or mitigation) should be avoided.

\subsection{Flow diagrams for disruption handling}

Our approach can be understood with the help of its envisaged schematic flow diagrams. The outer part of the controller (figure 1) has the task of identifying the proximity to the stability boundaries for all disruption paths by using all available sensors and plasma-state informations. Once a $p a t h_{i}$ is identified by its sensors, $\Sigma_{j}$ sensor $_{i, j}$, the corresponding handler, handler $_{i}$ (figure 2), is activated. The $\Sigma_{j}$ denotes a combination of all relevant sensors to describe the plasma state and generate a trigger. This primary selection within a supervisor potentially has to make a first prioritization between handlers, if the plasma gets close to multiple boundaries.

If during the activity of this handler $r_{i}$ another path $_{j}, j \neq i$ becomes a larger threat, the supervisor must be able to interrupt the active handler $_{i}$ and either switch to handler ${ }_{j}$, or combine their activity. If actuators are requested from multiple handlers, actuator management becomes a necessity. Since generally actuators can be used for different control goals within the control system, the handler will not have direct access to the actuator. For example the actuator "co-ECCD at the $q=2$ surface" does not imply control over gyrotrons, but only a request to the control system.

Each handler again uses its sensors, $\Sigma_{j}$ sensor $_{i, j}$, to continuously decide the appropriate action, i.e. recovery, avoidance or mitigation. At every time step, this decision must be reconsidered and allow for a change in the strategy, i.e. switching between recovery and avoidance. The irreversible mitigation should only be initiated by the handler, but then executed by the outer part of the control system. In ITER the mitigation will be performed by the Disruption Mitigation System (DMS) within the Central Interlock System (CIS). The DMS injects a situation dependent amount of material [43].

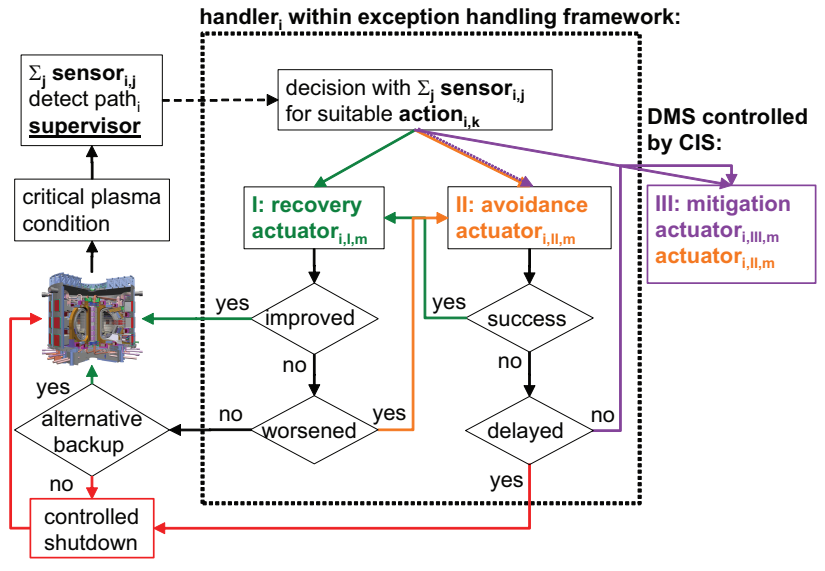

Figure 2. Flow diagram of the inner loop, the handler $_{i}$, which has to be defined for each disruption path $_{i}$ individually. The dotted rectangle defines the handler boundary.

The handlers should have one entry point and one interface for receiving commands for termination of its action or sharing resources (actuators). The handler provides four exit states:

(i) back to normal operation (successful recovery),

(ii) continuation with reduced demand (recovery not fully successful),

(iii) initiation of controlled rampdown (avoidance could only delay disruption) and

(iv) initiation of mitigation (avoidance failed or disruption inevitable ab initio).

For high $\beta_{N}$ disruptions, the conceptual structure will now be illustrated. In recovery mode the vanishing of the (3/2)-NTM and a recovery of $\beta_{N}$ is a successful exit condition (i). Remaining with low mode amplitude or reduced $\beta_{N}$ gives exit condition (ii). The avoidance scheme is initiated with a higher priority by the more dangerous $(m \geq 2 / n=1)$-mode or directly by an already locked $n=1$ mode. The unlocking and removal of a $(m \geq 2 / n=1)$-mode lets the handler switch internally from avoidance to recovery, in recovery mode the additional excitation of a $(m \geq 2 / n=1)$-mode would witch from recovery to avoidance. If the $(m \geq 2 / n=1)$ mode can not be removed, the condition (iii) for a controlled shutdown is given back. Should an $n=1$ locked mode become too large, mitigation would be initiated with condition (iv). The $n=2$, the $(m \geq 2 / n=1)$ and the locked mode amplitude from the magnetics, which will also be available at ITER, are the sensors which should be needed for these judgments.

In terms of actuator management, the MHD controller at AUG has implemented a scheme with a prioritization of the required actions for the application of central ECRH for avoiding core impurity and radiation peaking and the removal of rotating (2/1)NTMs and (3/2)-NTMs [45, 46, 47]. The MHD 
controller calculates the required radial deposition location for ECRH or ECCD and its power, depending on the occurrence of radiation peaking, $(3 / 2)$ or $(2 / 1)$ NTMs. This does not yet include the described disruption considerations.

Similar approaches are recently performed at TCV including also profile control [48]. In previous experiments the flexible ECRH system at TCV was used for combined removal and preemption of NTMs, while simultaneously controlling the sawtooth stability at the $q=1$ surface [26].

\section{Impurity induced disruption on TCV}

Experiments for disruption avoidance at TCV have been performed successfully in $\mathrm{H}$-modes which were generated by NBI and central ECRH heating. The toroidal field was selected such that the ECCD can reach the $q=2$ surface, both for avoidance or recovery without cutoff. This is in particular an issue for TCV with its low gyrotron frequency of $82.7 \mathrm{GHz}$ and the correspondingly low toroidal field to achieve a resonance inside the plasma.

With the application of the NBI and the resulting $\mathrm{L}-\mathrm{H}$ transition, a short phase of a rotating $n=1$ mode occurs, which immediately locks. This is due to momentum balance between the externally applied NBI torque and the intrinsic plasma rotation torque. The MHD degrades the H-mode confinement, but does not lead to a disruption. A disruption was later artificially caused by a pre-programmed neon puff to mimic uncontrolled influx of impurities of abnormal invessel failures. In figure 3, a discharge with successful disruption avoidance after such an injection is shown.

The increased radiated power due to the impurities was used as trigger. The appropriate sensor, the broadbanded bolometry measurement, was not available in real time and has been approximated by a sum over several central lines of sight of a soft X-ray array. An empirically defined threshold on this real-time signal was used. As a general trigger the soft X-ray measurements are not an appropriate choice, as this radiation is governed by the impurity content, the impurity species and the electron temperature. On top, in this case the soft X-ray measurement would have already triggered earlier in the L-mode. Considering the off-line calculated total radiation from bolometry (last trace in figure 3), a threshold applied to such a signal could serve as trigger. The radiation power after the Ne puff is not reached in normal non-disruptive discharges. Here the local ECCD is the actuator of an avoidance action within the handler, handler $r_{i m p-e v e n t}$, which is initiated by the radiation rise as sensor.

A figure of merit for this approach is the applied ECCD pulse length, $\Delta t_{E C}$, before either safe discharge

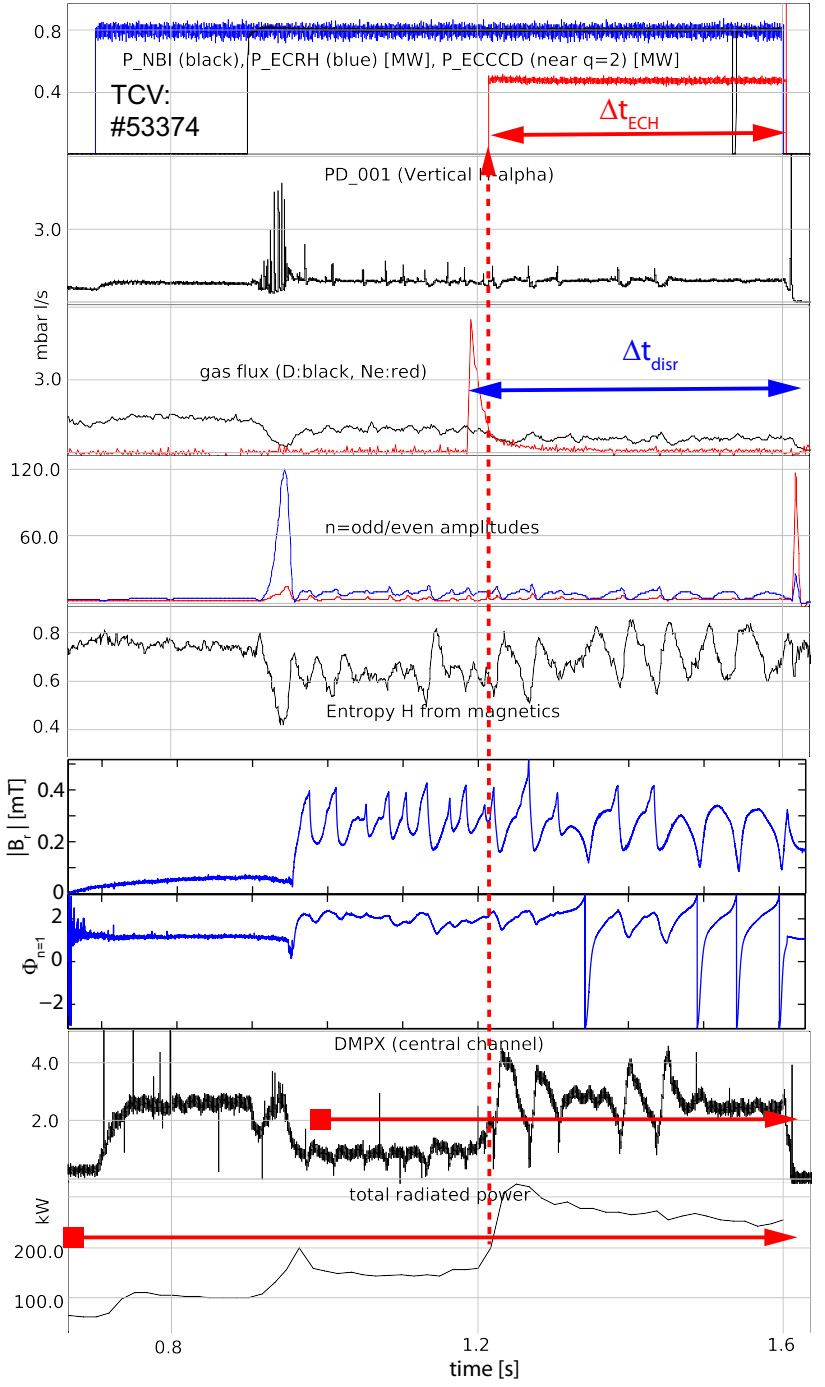

Figure 3. Time traces of the successfully recovered discharge $\$ 53374$. From top to bottom the time traces show: the applied central ECRH and NBI heating together with the triggered ECCD at the $q=2$ surface, the $H_{\alpha}$ emission in the divertor, the deuterium gas puff together with the neon puff, the $n=1$ and $n=2$ mode amplitudes, the entropy $\mathrm{H}$ from the SVD, the amplitude and the phase of the locked mode, a central line of sight of the soft X-ray measurement and the total radiated power measured from bolometry. The duration of the ECCD pulse, $\Delta_{E C H}$, and the time between the neon puff and the final disruption, after the ECCD has been switched off.

termination or a final disruption occurs. Varying the ECCD deposition radius, $\rho_{\text {dep }}^{E C D}$, showed that ECCD has to be localized around the $q=2$ surface to achieve disruption avoidance for the ECCD pulse length. This can be understood in terms of the $n=1$ locked mode that has a dominant $m=2$ component on this surface. The average mode amplitude is reduced during the ECCD phase, and hence the mode is unlocking and can make several revolutions. In the disruptive reference case without ECCD the locked mode amplitude is further growing and ultimately leads to the disruption. 

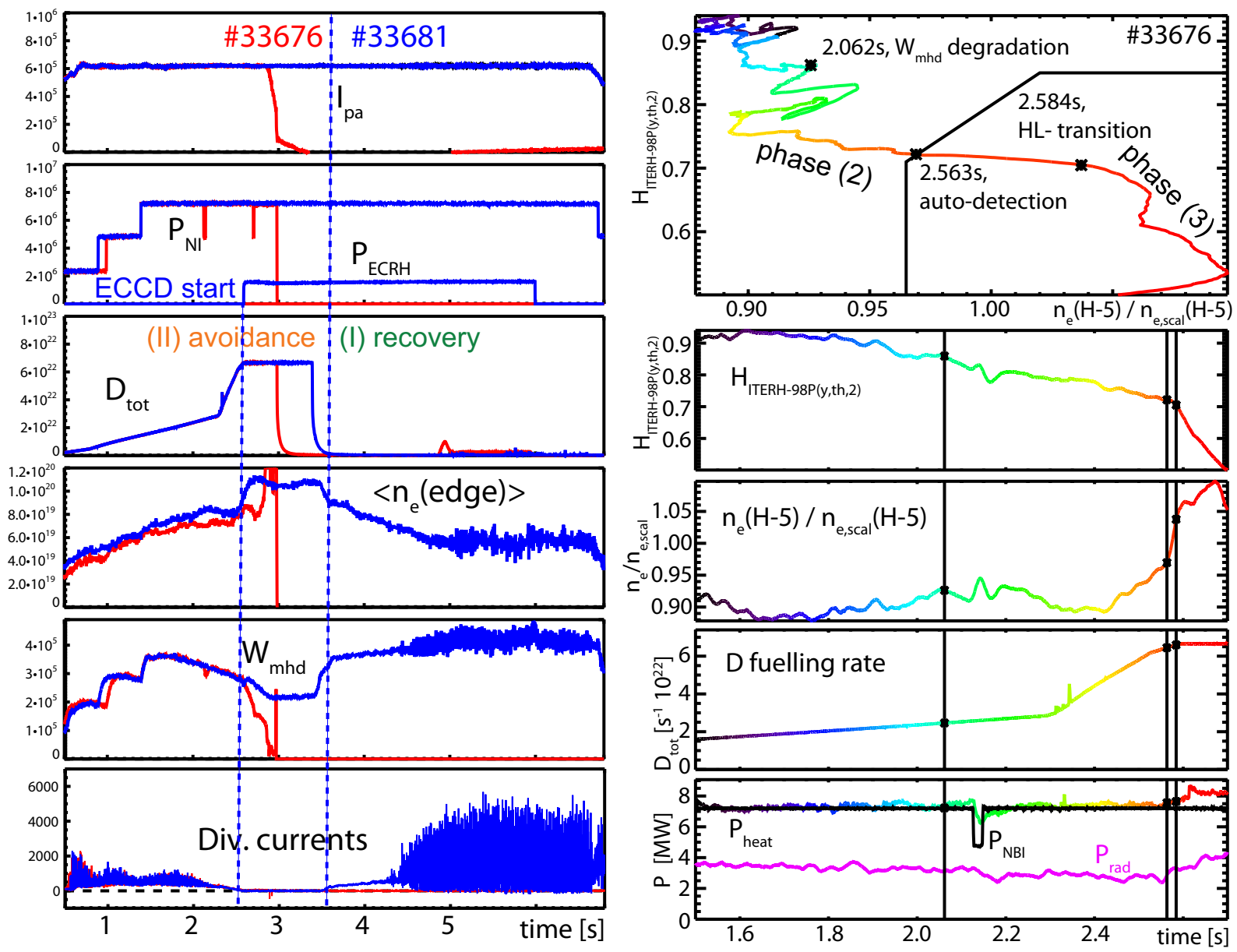

Figure 4. Left figure: Time traces of a disrupting reference density limit discharge \#33676 and discharge \#33681, where the disruption could be avoided with the application off ECCD and the confinement could be recovered by removing the strong gas puff. The traces show the plasma current, $I_{p a}$, the NBI heating power, $P_{N B I}$, and the applied ECCD, $P_{E C R H}$, the feed forward gas puff rate, $D_{t o t}$, the stored energy, $W_{m h d}$, and currents in the outer divertor. Right figure: For the disrupting discharge the trajectory in terms of $n_{e}(\mathrm{H}-5) / n_{e, \text { scal }}(\mathrm{H}-5)-H_{I T E R H-98 P(y, t h, 2)}$ is shown. The time is coded in rainbow colours. For orientation below the traces of $H_{I T E R H-98 P(y, t h, 2)}, n_{e}(\mathrm{H}-5) / n_{e, s c a l}(\mathrm{H}-5), D_{t o t}, P_{h e a t}, P_{N B I}$ and total radiated power $P_{\text {rad }}$ as function of time are shown.

\section{H-mode density limit}

In the $\mathrm{H}$-mode DL edge cooling first leads to an $\mathrm{H}$ $\mathrm{L}$ transition and finally to a DL disruption in Lmode $[7,8,9]$. The disruption is usually preceded by current profile peaking and $n=1 \quad(m=2$ or $m=3)$ modes which lock very quickly to the wall. Suitable actuators for path $h_{H D L}$ are ECCD in the vicinity of the $q=2$ rational surface and the control over the gas influx. However, the established disruption sensors at AUG (loop voltage rise, LM detector and amplitude of $n=1$ rotating mode) react deep in the L-mode phase, which turned out to be too late to avoid the disruption. Moreover, for ITER and beyond, the goal is also to sustain the H-mode. Therefore a trigger before the $\mathrm{H}$ $\mathrm{L}$ transition is required.

In [9] the H-mode DL is analyzed in terms of the stored energy $W_{m h d}$ and the electron density $\overline{n_{e}}$ of a peripheral line of sight of the DCN interferometer (channel H-5, with $\left.\rho_{p} \geq 0.7 \ldots 0.8\right)$, i.e. in an $\overline{n_{e}}\left(\rho_{p} \geq\right.$ $0.8)$ - $W_{m h d}$ plane. Four characteristic phases can be distinguished, which represent the sequence of events on path $_{H D L}$ : (1) Stable H-mode with constant plasma edge pressure and good confinement, (2) Degrading $H$-mode confinement with a significant change in the pedestal density profile shape, i.e. a rise in $\overline{n_{e}}\left(\rho_{p} \geq\right.$ 0.8 ), and a reduction of the edge pressure, associated with a reduction in the stored energy, $W_{m h d}$, (3) Breakdown of the H-mode, i.e. transition to low confinement L-mode with a fast reduction of $W_{m h d}$ at roughly constant edge density and (4) L-mode disruption sequence with MHD activity. For AUG a scaling for the onset of phase (3), i.e. the high-density induced H-L transition, is determined for a wide range of heating power $\left(6 \mathrm{MW} \leq P_{\text {heat }} \leq 12.5 \mathrm{MW}\right)$, safety factor $\left(3.5 \leq q_{95} \leq 6\right)$, plasma current $(0.6 \mathrm{MA} \leq$ 
$\left.I_{p} \leq 1.2 \mathrm{MA}\right)$, toroidal magnetic field $\left(1.45 \mathrm{~T} \leq B_{t} \leq\right.$ $2.7 \mathrm{~T})$ and triangularity $(0.23 \leq \delta \leq 0.37)[9]$ :

$\overline{n_{e, s c a l}}(\mathrm{H}-5)=(0.506 \pm 0.192) \frac{P_{\text {heat }}^{0.396 \pm 0.13} I_{p}^{0.265 \pm 0.14}}{q_{95}^{0.323 \pm 0.14}}$

where $I_{p}$ is given in MA, $P_{\text {heat }}$ in MW and $\overline{n_{e, s c a l}}$ in $10^{20} \mathrm{~m}^{-2}$. To achieve this scaling, the discharge conditions were carefully kept constant and the gas puff increase was slow.

\subsection{Experiments at ASDEX Upgrade}

To study the effectiveness of the actuators, experiments were carried out in reproducible discharges $\left(I_{p}=0.6\right.$ MA, $B_{t}=-2.5 \mathrm{~T}$ with pre-programmed gas puff ramp), which showed the H-L transition at nearly the same time. ECCD in the vicinity of $q=2$ was activated pre-programmed at the expected H-L transition onset, which led to an ECCD start (10-50 ms) after the actual H-L transition, while keeping the gas puff rate high. The left part of figure 4 compares a disrupting discharge without ECCD (red traces) and an example for disruption avoidance using ECCD. The latter discharge remained stable at even higher densities compared to those reached in the disrupting one. A subsequent reduction of the gas puff rate recovers the $\mathrm{H}$-mode with high confinement, albeit at reduced density. Activation of the ECCD only $100 \mathrm{~ms}$ later fails to avoid the disruption (see figure 7 , orange trajectory).

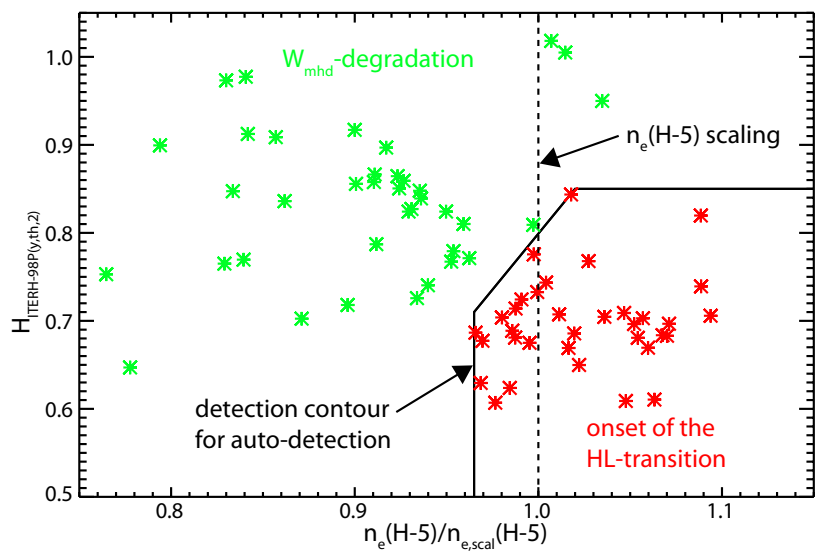

Figure 5. Operation diagram in terms of $n_{e}(\mathrm{H}-5) / n_{e, s c a l}(\mathrm{H}-5)$ versus $H_{I T E R H-98 P(y, t h, 2)}$. The green points represent the times when the discharge performance is already degrading in terms of $H_{I T E R H-98 P(y, t h, 2)}$ (phase $\left.(2)\right)$, the red points show the time of the start of the H-L transition (phase (3)). The solid black curve represents the suggested operation boundary at which the disruption handler should be activated.

These experiments demonstrate that a trigger for handler $H D L$ is required reliably before the H-L transition to allow avoidance of this disruption type at AUG. For application to a variety of plasma scenarios,

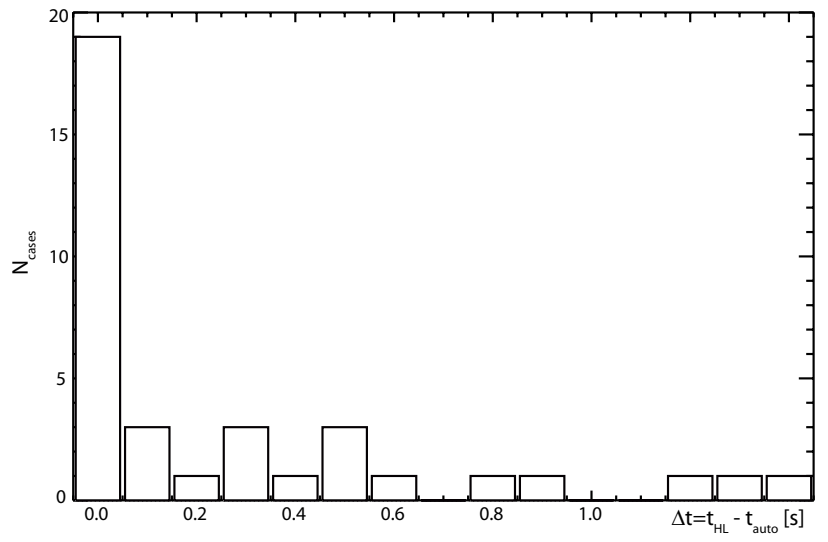

Figure 6. Distribution of the time delay between the manual detection of the H-L transition, $t_{H L}$, and the automatic detection, $t_{\text {auto }}$.

the trigger-relevant quantities should be dimensionless. We therefore normalize the electron density to the scaling in equation (1), $f_{H L}=\overline{n_{e}}(\mathrm{H}-5) / \overline{n_{e, s c a l}}(\mathrm{H}-5)$, and replace the stored energy, $W_{m h d}$, by the H-factor, $H_{98}$, of the ITER-98P(y,th,2) scaling (equation (20) in [49]). On the right hand side of figure 4, top box, the trajectory of the disrupting case (left part of this figure, red traces) in the $f_{H L}-H_{98 P}$ plane reveals similar behaviour as the discharges analyzed in [9]. The onset of phase (3) and a point within the early phase (2) are indicated.

The discharges in the database from [9] and all disruption avoidance attempts were reinspected in the $f_{H L}-H_{98 P}$ plane. For each discharge the onset of the $\mathrm{H}-\mathrm{L}$ transition, $t_{H L}$, and a time point when the $\mathrm{H}-$ mode confinement is already degrading are identified. The latter does not necessarily represent the onset of phase (2), since this cannot always be clearly identified in discharges with steeper gas puff ramp. Figure 5 shows these time points in the $f_{H L}-H_{98}$ plane. In this plane we can define an empirical boundary that separates the two sets of points. The crossing of this boundary from the "safe" to the "critical" region (lower right) - after H-mode had been reached for the first time within the discharge - might be used as an automatic trigger, $t_{\text {auto }}$, for starting handler h $_{H L}$. The distribution of the time difference $\Delta t=t_{H L}-$ $t_{\text {auto }}$ between the manually detected onset of the $\mathrm{H}-\mathrm{L}$ transition and the first entering of the "critical" region (see figure 6) has a clear maximum around $0.1 \mathrm{~s}$. Thus, an automatic trigger based on the proposed boundary is in most cases only shortly before the H-L transition, which makes it a promising candidate for a sensor within handler $H D L$.

Figure 7 shows the trajectories in the $f_{H L}$ $H_{98}$ plane of the reference disrupting discharge (\#33676,red), the safely avoided disruption case at high $n_{e}$ (\#33681, blue) and a discharge where the 
ECCD was applied $100 \mathrm{~ms}$ later which did not prevent the disruption (\#33678, orange).

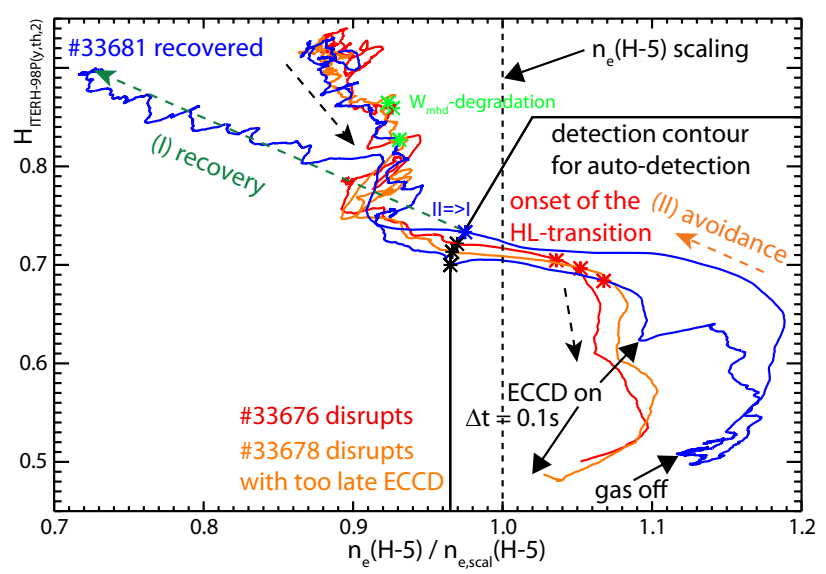

Figure 7. Operation diagram in terms of $n_{e}(\mathrm{H}-5) / n_{e, s c a l}(\mathrm{H}-5)$ versus $H_{I T E R H-98 P(y, t h, 2)}$ with trajectories from the disrupting reference discharge \#33676, the saved and recovered discharge \#33681 and discharge \#33678, where the ECCD has been applied too late and the discharge disrupts (see figure 4). In all cases the proposed trigger threshold would have been early enough to safely recover the discharge.

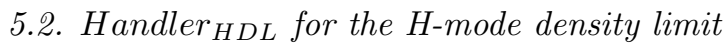

For an application in a plasma with significant $\alpha$ particle heating further considerations are required. The total heating power becomes $P_{\text {heat }}=P_{\text {heat } \text { ext }}+$ $0.2 \cdot P_{f u s}$, with the fusion power, $P_{f u s} \sim \beta_{N}^{2}$, and the external heating power, $P_{\text {heat,ext }}$. For a reduction in $P_{f u s}$ it follows with equation (1), that the reachable density, $\overline{n_{e, s c a l}} \sim P_{\text {heat }}^{0.396}$ gets reduced and in figure 5 the operation point moves towards higher $f_{H L}=$ $\overline{n_{e}} / \overline{n_{e, s c a l}}$. The confinement loss in the degrading $\mathrm{H}-$ mode (phase (2)), but surely the degradation to Lmode (phase (3)) will be intolerable for disruption avoidance or recovery. A ramp-down of the discharge would be the only available option.

Within the proposed framework the handler, handler $_{H D L}$, for the H-mode density limit might look as follows. Once the plasma state crosses the operational boundary in the $f_{H L}-H_{98 P}$ plane, the handler is started from the outer loop and takes control over two actuators, ECCD and gas influx. As sensors it needs the plasma state in the $f_{H L}-H_{98 P}$ plane, a MARFE detector and the LM detector. The handler starts with avoidance action by adding ECCD and reducing the gas puff. The density reduction must be controlled such that the discharge still generates sufficient fusion power, $P_{\text {fus }}$. In parallel, the MARFE detector can initiate a controlled shut down (exit state (iii)) and the LM detector can trigger mitigation (exit state (iv)). If the avoidance is successful, and the "critical" region in the $f_{H L}-H_{98 P}$ plane is left, the handler switches from avoidance to recovery $(\approx 3.539 \mathrm{~s}$ of \#33681 on the left part of figure 4 and the blue point (II => I) in figure 7) with the aim of further confinement improvement. The point at which the discharge is given back to the outer control system and the decision of the exit state for the successfully avoided disruptions ((i) or (ii)), is not yet specified.

ECCD at the $q=2$ surface, will be available within the ECRH and ECCD system at ITER for MHD control [50], and the control system can modify the gas influx. The detailed strategy of actuator application might differ in an $\alpha$-particle heated plasma and can be modeled in the future [51]. For the normal discharge termination the avoidance of a density limit disruption is also an issue [52].

Most of the required parameters for generating the plasma state in the $f_{H L}-H_{98 P}$ plane $\left(I_{p}, P_{\text {heat }}\right.$, $\left.P_{\text {rad }}, B_{t}, a, R_{g e o}, \kappa,\left\langle n_{e}\right\rangle, M\right)$ are reliably available in real time at AUG. The most critical value is the interferometer density measurement $\left(\left\langle n_{e}\right\rangle\left(\rho_{p} \geq 0.8\right)\right)$ which can be corrupt when fringe jumps occur. This could be replaced by the recently available density observer within the RAPTOR observer, which can provide density profiles even under difficult conditions $[53,54,55]$. At any future experiment the above parameters will be needed in the control system or can be easily generated.

\section{Summary and outlook}

In ITER and DEMO the requirements on disruption managment are more demanding than in present devices [1]. Mitigated disruptions should be restricted to major failures, and premature controlled discharge terminations should only be executed when no recovery to a useful scenario is technically feasible. A concept for handling approaching disruptions with these constraints has been proposed. Within this concept, actions for recovery, avoidance and mitigation, depending on both disruption path and discharge condition, have to be developed and tested in present-day tokamaks. For each disruption path a handler, consisting of sensors, triggers, actuators and its algorithm, must be designed. Unlike common generic disruption triggers, which occur typically only shortly before the disruption, the path-specific handlers can allow for earlier intervention and thus offer a greater flexibility and higher chance of full discharge recovery. Sensors can be combined and considered within a general plasma-state observer, actions and in particular actuators have to be managed in case of competing requirements.

Experiments on impurity puffed H-mode disruptions at TCV and the H-mode density limit at ASDEX Upgrade were presented. In both cases, ECCD 
in the vicinity of the $q=2$ surface was applied successfully as actuator to avoid the disruption or even recover the discharge. For the H-mode density limit, ECCD was activated feed forward together with the control over the gas influx as second actuator, as the conventional, MHD based disruption sensors trigger too late. For the $\operatorname{path}_{H D L}$ a new plasma-state sensor was developed, which provides a sufficiently early trigger. With the newly available reliable real-time density signal from the RAPTOR suite at ASDEX Upgrade, this plasma-state sensor will be applied in the coming experiments in 2017. In the future the developed plasmastate based trigger requires further off-line verification and real-time application.

For the application in an $\alpha$-particle heated plasma, further contraints have to be taken into account for the application of actuators to achieve the control goals. At any time the released fusion power, $P_{f u s}$, generating the internal heating, has be maintained.

In order to implement the proposed concept as a real scheme the following steps should be performed.

1: create or use a statistical data analysis for the most likely root cause of disruptions and describe its path $_{i}$ towards disruption

2: identify appropriate sensors, sensor $_{i, j}$ for each relevant path $_{i}$

3: identify appropriate actuators for recovery (I), avoidance (II) and mitigation (III) of each path, i.e. actuator $_{i, I, k}$, actuator $_{i, I I, k}$ and actuator $_{i, I I I, k}$

4: implement the specific handler, handler h $_{i}$ for that path, which uses these actuators and sensors

5: repeat step 2-4 for all relevant paths from 1

6: create a supervisor managing the handlers including possibly actuator management

\section{Acknowledgment}

This work has been carried out within the framework of the EUROfusion Consortium and has received funding from the Euratom research and training programme 2014-2018 under grant agreement No 633053. The views and opinions expressed herein do not necessarily reflect those of the European Commission.

\section{References}

[1] Lehnen, M. and Aleynikov, P. E. A., in Proc. of the 26th IAEA Conference Fusion Energy (CD-Rom), Kyoto, Japan, October 2016, volume 0, pages IAEA$\mathrm{CN}-234 / \mathrm{EX} / \mathrm{P} 6-39$.

[2] LEHNEN, M. et al., Journal of Nuclear Materials 463 (2015) 39

[3] DE VRIES, P. C. et al., Nuclear Fusion 51 (2011) 053018.

[4] DE VRIES, P. C. et al., Plasma Physics and Controlled Fusion 21 (2014) 056101.
[5] PAUtasso, G. et al., in Europhysics Conference Abstracts (CD-ROM, Proc. of the 41th EPS Conference on Plasma Physics, Berlin, Germany, 2014), volume $38 \mathrm{~F}$ of $E C A$, page $\mathrm{P} 2.015$.

[6] MERTENS, V. et al., Plasma Physics and Controlled Fusion 36 (1994) 1307.

[7] MERTENS, V. et al., Nuclear Fusion 37 (1997) 1607.

[8] MERTENS, V. et al., Nuclear Fusion 40 (2000) 1839.

[9] BERNERT, M. et al., Plasma Physics and Controlled Fusion 57 (2015) 014038.

[10] NEU, R. et al., Journal of Nuclear Materials 438 Supplement (2013) S34.

[11] NEU, R. et al., Plasma Science, IEEE Transactions on 42 (2014) 552.

[12] CIANFARANI, C. et al., in Europhysics Conference Abstracts (CD-ROM, Proc. of the 40th EPS Conference on Plasma Physics, Espoo, Finland, 2013), volume 37D of ECA, page P5.165.

[13] GALPERTI, C. et al., Plasma Physics and Controlled Fusion 56 (2014) 114012.

[14] REICH, M. et al., Fusion Science and Technology 61 (2012) 309.

[15] MARASCHEK, M. et al., in Europhysics Conference Abstracts (CD-ROM, Proc. of the 40th EPS Conference on Plasma Physics, Espoo, Finland, 2013), volume 37D of $E C A$, page $\mathrm{P} 4.127$.

[16] BERNERT, M. et al., Review of Scientific Instruments 85 (2014) 033503.

[17] MERTENS, V. et al., Fusion Science and Technology 44 (2003) 593.

[18] FELICI, F. et al., in Proc. of the 26th IAEA Conference Fusion Energy (CD-Rom), Kyoto, Japan, October 2016, volume 0, pages IAEA-CN-234/EX/P8-33.

[19] BERKERY, J. W. et al., Physics of Plasmas 24 (2017) 056103.

[20] BERKERY, J. W. et al., in Europhysics Conference Abstracts (CD-ROM, Proc. of the 44th EPS Conference on Plasma Physics, Belfast, UK, 2017), volume 41F of ECA, page P1.138.

[21] ESPOSITO, B. et al., Nuclear Fusion 49 (2009) 065014.

[22] ESPOSITO, B. et al., Nuclear Fusion 51 (2011) 083051.

[23] MARASCHEK, M., in Europhysics Conference Abstracts (CD-ROM, Proc. of the 42th EPS Conference on Plasma Physics, Lisbon, Portugal, 2015), volume 39E of ECA, page P1.112.

[24] REICH, M. et al., in Europhysics Conference Abstracts (CD-ROM, Proc. of the 40th EPS Conference on Plasma Physics, Espoo, Finland, 2013), volume 37D of ECA, page P2.151.

[25] PETTY, C. C. et al., Nuclear Fusion 44 (2004) 243.

[26] FELICI, F. et al., Nuclear Fusion 52 (2012) 074001.

[27] VOLPE, F. A. G. et al., Physics of Plasmas 16 (2009) 102502.

[28] VOLPE, F. A. et al., Physical Review Letters 115 (2015) 175002.

[29] PACCAGNELLA, R., in Europhysics Conference Abstracts (CD-ROM, Proc. of the 43th EPS Conference on Plasma Physics, Leuven, Belgium, 2016), volume 40A of ECA, page P1.027.

[30] GRANUCCI, G., in Europhysics Conference Abstracts (CD-ROM, Proc. of the 42th EPS Conference on Plasma Physics, Lisbon, Portugal, 2015), volume 39E of ECA, page P1.108.

[31] KULKARNI, S. et al., in Proc. of the 25th IAEA Conference Fusion Energy (CD-Rom), St. Petersburg, Russian Federation, October 2014, volume IAEA-CN221, pages EX/P7-16.

[32] STOBER, J. et al., Nuclear Fusion 43 (2003) 1265.

[33] PAUTASSO, G., in Europhysics Conference Abstracts (CD-ROM, Proc. of the 42th EPS Conference on Plasma 
Physics, Lisbon, Portugal, 2015), volume 39E of ECA, page P1.134.

[34] PAUTASSO, G. et al., Nuclear Fusion 47 (2007) 900.

[35] PAUTASSO, G. et al., Nuclear Fusion 36 (1996) 1291.

[36] PAUtASSO, G. and GRUBER, O., Fusion Science and Technology 44 (2003) 716.

[37] COMMAUX, N. et al., Nuclear Fusion 50 (2010) 112001.

[38] PENAFLOR, B. G. et al., Fusion Engineering and Design 84 (2009) 1484.

[39] FERRON, J. R. et al., Fusion Engeneering 2 (1995) 870.

[40] SAMMULI, B. S. et al., Fusion Engineering and Design 85 (2010) 456.

[41] HUMPHREYS, D. A. et al., Physics of Plasmas 22 (2015) 021806.

[42] RAUPP, G. et al., Fusion Engineering and Design 89 (2014) 523.

[43] RAUPP, G. et al., Fusion Engineering and Design $\mathbf{x x x}$ (2017) in press.

[44] EIDITIES, N. W. et al., in Proc. of the 57th Annual APS Meeting of the Division of Plasma Physics, Savannah, USA, 2015.

[45] RAPSON, C. J. et al., in 2016 41st International Conference on Infrared, Millimeter, and Terahertz waves (IRMMW-THz), pages 1-2, 2016.

[46] RAPSON, C. J. et al., Fusion Engineering and Design In press, corrected proof (2017) .

[47] RAPSON, C. et al., Nuclear Fusion 57 (2017) 076023.

[48] KONG, M. et al., in Proc. of 44th EPS Conf. on Plasma Phys., Belfast, UK, 2017, page P4.152.

[49] ITER Physics Expert Groups on Confinement and Transport and Confinement Modelling and Database, Nuclear Fusion 39 (1999) 2175.

[50] HENDERSON, M. et al., Nuclear Fusion 48 (2008) 054013 (14pp).

[51] JANKY, F. et al., Fusion Engineering and Design $\mathbf{x x x}$ (2017) in press.

[52] DE VRIES, P. C. et al., in Proc. of 44th EPS Conf. on Plasma Phys., Belfast, UK, 2017, page O4.119.

[53] KUDLACEK, O. et al., in Proc. of 44th EPS Conf. on Plasma Phys., Belfast, UK, 2017, page P2.162.

[54] FELICI, F. et al., in Proc. of 44th EPS Conf. on Plasma Phys., Belfast, UK, 2017, page P1.151.

[55] BLANKEN, T., in Europhysics Conference Abstracts (CDROM, Proc. of the 43th EPS Conference on Plasma Physics, Leuven, Belgium, 2016), volume 40A of ECA, page P4.031. 